\title{
Mechanism of blood-retinal barrier breakdown induced by HIV-1 (Review)
}

\author{
$\mathrm{XIN} \mathrm{CHE}^{1,2}, \mathrm{XIAN}-\mathrm{QUN}$ FAN $^{1}$ and ZHI-LIANG WANG ${ }^{1}$ \\ ${ }^{1}$ Department of Ophthalmology; ${ }^{2}$ Key Laboratory of Ophthamology, \\ Ninth People's Hospital Affiliated with Shanghai Jiaotong University, Shanghai 200011, P.R. China
}

Received September 23, 2013; Accepted January 28, 2014

DOI: $10.3892 /$ etm.2014.1521

\begin{abstract}
Human immunodeficiency virus (HIV)-1 has been detected in ocular tissues; however, the mechanism of entry has not been established. It has been hypothesized that the blood-retinal barrier (BRB), a critical guardian against microbial invasion of the eye, may be compromised in the presence of HIV-1 in the eye. In vivo and in vitro model systems have shown that the breach of tight junctions induced by HIV-1-associated factors contributes to the breakdown of the BRB. The present study reviews the mechanism of tight junction disruption, focusing on signaling pathways, the expression of enzymes, including metalloproteinases, and cytokines that affect inflammation. The studied pathways may be potential targets for the prevention of ocular HIV complications.
\end{abstract}

\section{Contents}

1. Introduction

2. Components of the blood-retinal barrier

3. Tight junctions in the eye

4. Claudins

5. Occludin

6. Zonula occludens-1

7. Mechanism for the disruption of tight junctions

8. Tat-induced caveolae-associated signaling

9. Disruption of tight junctions and the basal lamina by secreted matrix metalloproteinases

10. Disruption of tight junctions by alterations in the actin cytoskeleton

11. Inflammatory cytokines induce the destruction of tight junctions

12. Concluding remarks

Correspondence to: Professor Zhi-Liang Wang, Department of Ophthalmology, Ninth People's Hospital Affiliated with Shanghai Jiaotong University, 639 Zhizaoju Street, Shanghai 200011, P.R. China E-mail: ophthalmologycc@163.com

Key words: actin cytoskeleton, blood-retinal barrer, caveolin-1, matrix metalloproteinases, ocular HIV-1, tight junction

\section{Introduction}

Han et al performed a cross-sectional study on a group of human immunodeficiency virus (HIV)-1-infected patients who underwent long-term highly active antiretroviral therapy. The results indicated that various fragments of the HIV-1 genome were detectable in tears, in the absence of a detectable plasma viral load (1). Earlier in the 1980s, studies isolated HIV viruses from tears, cornea, aqueous humor, conjunctiva, retinal vascular endothelium and even contact lenses (2-4). Pathanapitoon et al analyzed the aqueous and vitreous humor samples from HIV-1-infected patients and observed that several patients had intraocular HIV-1 RNA levels that were higher than the corresponding HIV-1 RNA plasma levels, which indicated a largely elevated ocular-to-plasma HIV ratio (5). Thus, the mechanisms by which HIV invades the eye and exists in the tissues in the absence of a detectable plasma virus level were questioned. To date, there has been no explanation of these circumstances. A growing number of studies have shown that the central nervous system (CNS) is a sanctuary for HIV, which crosses the blood-brain barrier (BBB) early in the course of systemic infection and resides in brain macrophages and microglia (6,7). One hypothesis is that HIV persists in these sanctuaries during antiretroviral treatment and may cause the generation and dissemination of drug-resistant viruses (8). Another hypothesis is that the breakdown of the blood-retinal barrier (BRB), which is associated with the changes in the tight junctions, contributes to the trafficking of HIV into the eye $(9,10)$. Therefore, the present review focused on the key breakdown mechanisms of tight junctions.

\section{Components of the blood-retinal barrier}

The BBB provides significant protection against microbial invasion of the brain (11). The BRB and BBB are derived from the same embryonic primordium. Brain endothelial cells form extremely tight cell-cell junctions that are distinct from the tight junctions of endothelia and epithelia elsewhere in the body. Brain endothelial cells lack fenestrations and have a high number of mitochondria, which are characteristics associated with their specialized functions. For example, a high mitochondrial content is likely to be important for providing the energy required to maintain the structure and function of the BBB (12). For BBB capillaries, the transendothelial elec- 
trical resistance, an indicator of permeability, ranges between 1,000 and $2,000 \Omega / \mathrm{cm}^{2}$. However, for systemic capillaries this value is only $5-10 \Omega / \mathrm{cm}^{2}$. The BRB, which maintains eye homeostasis, has a similar nature to the BBB (13). The BRB is composed of retinal capillary endothelial cells (inner BRB) and retinal pigment epithelium (RPE) cells (outer BRB) (14). These two cell types develop tight junctions that confer a high degree of control of solute and fluid permeability between the circulating blood and the neural retina (Fig. 1).

\section{Tight junctions in the eye}

The transmembrane proteins of tight junctions include occludin, junction adhesion molecules and claudins. These proteins extend into the paracellular space, acting in concert to affect barrier properties (15). Occludin and claudins have external loops that mediate intercellular adhesion by interaction with occludin and claudins of neighboring cells (16). In addition, claudins and occludin interact with zonula occludens (ZOs) $-1,-2$ and -3 , which in turn associate with the actin cytoskeleton (Fig. 2). The 220-kDa phosphoprotein ZO-1, in particular, is able to bind to a wide variety of protein partners and allow for the control of tight junction assembly (17). During viral infections and other pathological conditions, altering the localization or cleavage of the tight junction proteins is the main pathological change, which results in the increasing permeability of the barrier (18).

\section{Claudins}

Claudin-5 is expressed predominantly in endothelial cells (19). A study using claudin-5-deficient mice demonstrated that it is necessary to preserve the vascular barrier to small $(<0.8 \mathrm{kDa})$ molecules in the brain (20). As claudin-5 is expressed in the retinal vasculature (21), it is likely to contribute to the function of the BRB. The expression of claudin-3, -10 and -19 has been detected in the human fetal RPE (22).

\section{Occludin}

Increased expression of occludin has been observed to correlate with increased barrier function and decreased paracellular permeability $(23,24)$. In addition, changes in the content and localization of occludin in diabetic retinas have been demonstrated to be associated with alterations in barrier function (25). A study of bovine retinal endothelial cells treated with vascular endothelial growth factor (VEGF) revealed a decreased occludin content and immunostaining at cell borders concomitant with increased BRB permeability (26). Similarly, diabetes reduces occludin content in the brain vasculature; this reduction correlates with the incidence of vascular diseases (27).

\section{Zonula occludens-1}

$\mathrm{ZO}$ proteins are intracellular proteins that associate with the cytoplasmic surface of tight junctions and organize the tight junction complex. The presence of ZO-1 is readily observed in retinal vascular endothelial and RPE cells. In these cell types, agents that induce permeability, including VEGF or hepato- cyte growth factor, induce the redistribution of $\mathrm{ZO}-1$ from the cell border to the cell interior $(28,29)$.

\section{Mechanism for the disruption of tight junctions}

Increased permeability of the BRB may occur through two pathways, the paracellular or the transcellular pathway. The paracellular route is governed by tight junctions and is usually the main route of increased endothelial barrier permeability (30).

\section{Tat-induced caveolae-associated signaling}

Tat is the only protein actively secreted by HIV-1 infected cells. It circulates in the blood at high levels during HIV infection and crosses the $\mathrm{BBB}$ with large quantities entering the CNS (31). The VEGF receptor has been hypothesized to serve as a high-affinity receptor for Tat in endothelial cells (32). Tat specifically interacts with VEGF and surface molecules that belong to the large family of G-protein-coupled receptors localized to caveolae, to activate several protein kinases, including certain kinases involved in Ras signaling (33). Ras proteins are small GTPases that cycle between inactive GDP-bound and active GTP-bound conformations. Several elements of the Ras signaling cascades are localized in caveolae, the dominant type of lipid rafts in endothelial cells (34). Zhong et al focused on the breakdown mechanism of the BBB and found that Tat diminished the expression of several tight junction proteins, including occludin, ZO-1 and ZO-2, in the caveolar fraction of human brain microvascular endothelial cells (HBMECs) (35). These effects were effectively protected against by the pharmacological inhibition of Ras signaling and by the silencing of caveolin-1. Lin et al demonstrated that HIV infection in primary human monocyte-derived macrophages results in a marked upregulation of caveolin-1 expression mediated by the HIV Tat protein (36). Nag et al assessed the sequential expression of caveolae and occludin over a period of $12 \mathrm{~h}$ to 6 days post-lesion in a rat cortical cold injury model. The study demonstrated a significant increase in endothelial caveolin-1 expression in arterioles and large veins, particularly those with BBB breakdown to proteins (37). In addition, the HIV-1 Tat protein causes the paracellular permeability of RPE cells to increase in vitro, concomitant with changes in the expression of tight junctions. Therefore, the effects of Tat on the outer BRB may be mediated by Ras/ERK1/2 pathways (9).

\section{Disruption of tight junctions and the basal lamina by secreted matrix metalloproteinases}

The basal lamina of the BBB contains extracellular matrix molecules, including laminin, type IV collagen and fibronectin. The majority of these molecules are substrates for a family of neutral proteases called matrix metalloproteinases (MMPs), in particular MMP-2 and -9 (38). MMPs contribute to interactions between cells and the matrix, allowing movement and shape changes in CNS development and neuronal plasticity. MMPs are key mediators of tight junction protein alterations, which lead to BBB dysfunction $(39,40)$. These zinc-dependent enzymes have proteolytic activity that acts on the extracellular matrix, including the basal laminae. 


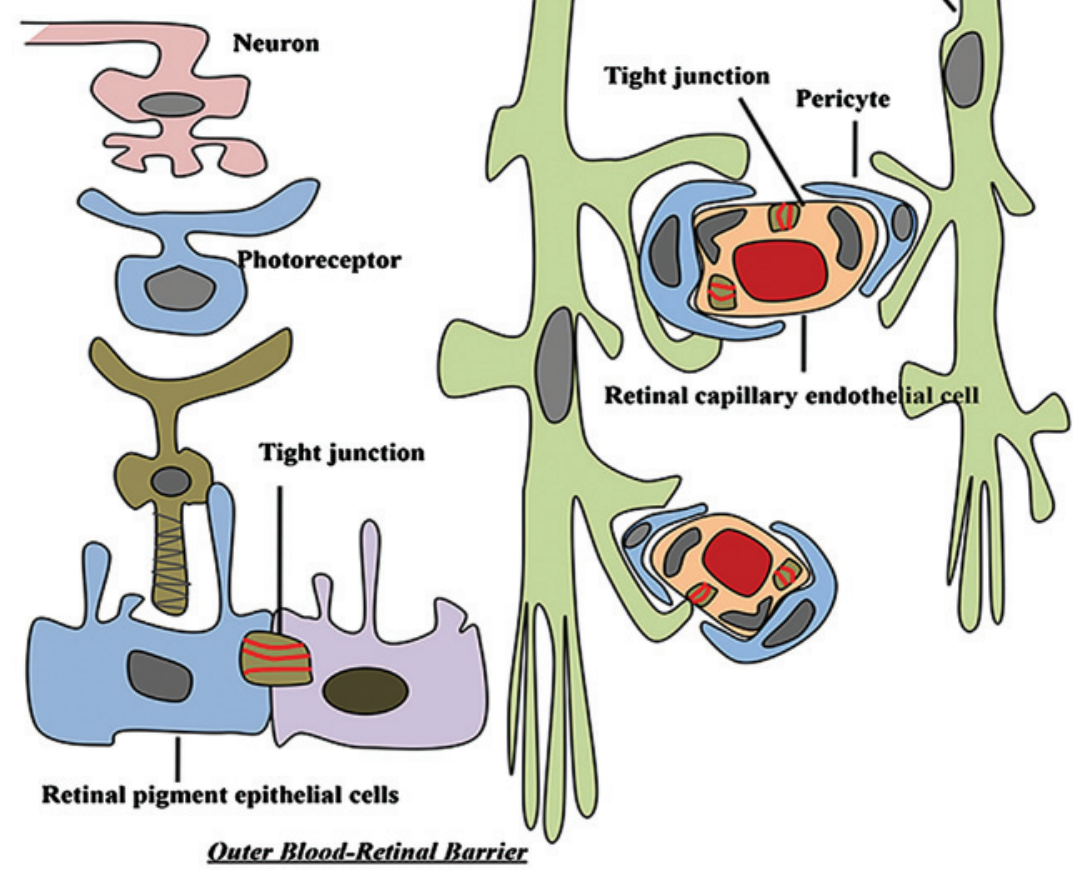

Figure 1. Retinal-vascular unit and tight junctions between endothelial cells, forming the inner and outer blood-retinal barrier

Blood

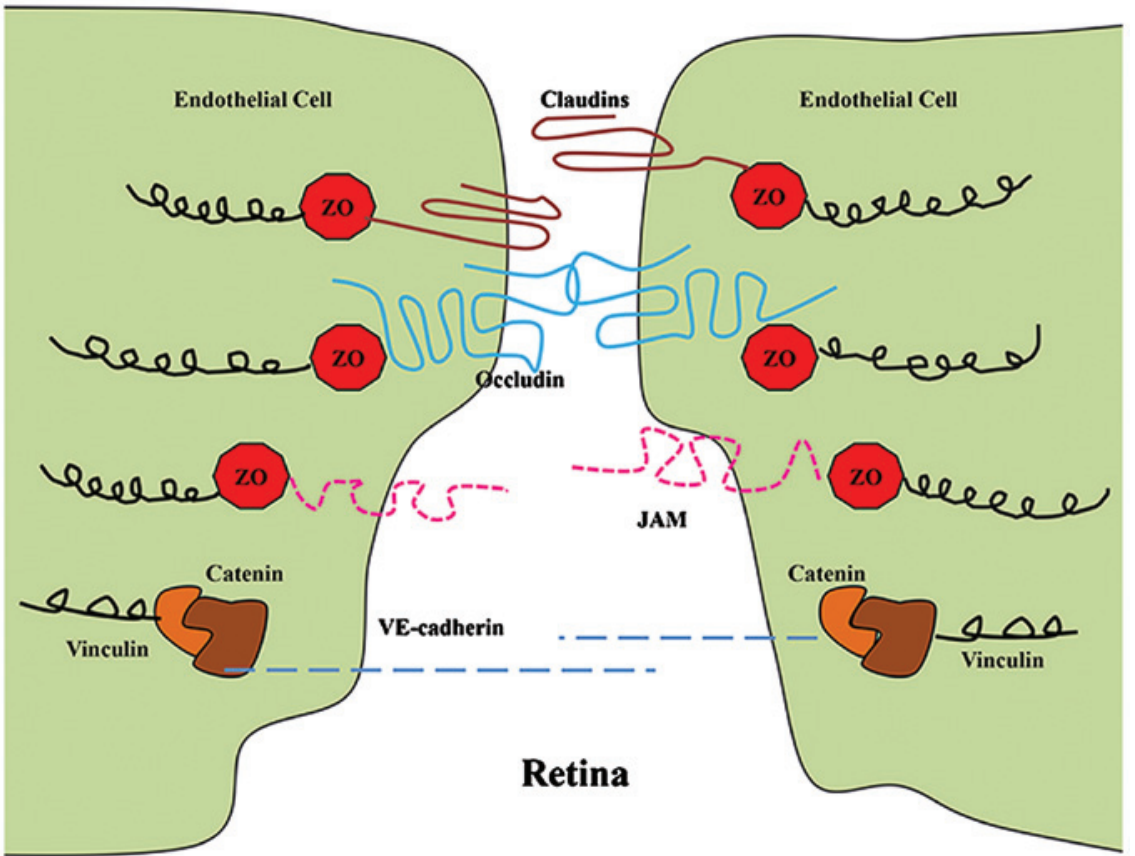

Figure 2. Major molecules of the tight and adherens junctions are shown. Tight junction proteins include ZO, occludin, claudins and JAMs, while adherens junction proteins include catenins and vinculins. JAMs, junctional adhesion molecules; ZO, zonula occludens.

MMPs are associated with tight junction disruption not only by basement membrane degradation, but also by cleavage of tight junction proteins $(41,42)$. Elevated levels of MMP-9 have been reported in the cerebrospinal fluid of HIV-1-infected children (43) and adult patients (44). The overexpression of MMP-2 and MMP-9 was also reported in the brain of a severe combined immunodeficiency mouse model of HIV-1 encephalitis (45). In a study in rats, within 30 min of HIV-1 glycoprotein 120 (gp120) injection into the caudate-putamen (CP), MMP-2 co-localized with laminin and by $6 \mathrm{~h}$ there was 
a significant reduction in the number of laminin-positive structures in the injected CP. Similarly, the levels of vascular tight junction proteins, claudin-5 and occludin, were significantly decreased in the experimental group compared with those in the controls (46). In one study, primary HBMECs were exposed to HIV-1 Tat proteins. Tat induced MMP-9 expression, and RNA interference targeting MMP-9 reduced the paracellular permeability of Tat-treated HBMECs and the concentration of soluble occludin in the cell supernatant (47). In a diabetic rat model, the transepithelial electrical resistance (TER) was measured in the retinal endothelium and RPE following treatment with MMPs. The two cell types showed decreased TER and degradation of the tight junction proteins, indicating that elevated expression levels of MMPs in the retina may facilitate the change in BRB permeability (48).

\section{Disruption of tight junctions by alterations in the actin cytoskeleton}

Tight junctions may also be disrupted from within cells. Changes in the actin cytoskeleton are likely to occur upon alteration of the tight junction proteins, resulting in paracellular permeability changes (49). Reactive oxygen species play a role in disrupting tight junctions from within cells via the induction of the RhoA small GTPase, phosphoinositide 3-kinase and protein kinase B signaling pathways, concomitant with the rearrangement of the actin cytoskeleton and altered localization of occludin and claudin-5 (50). In addition, the alteration of the actin cytoskeleton induced by hypoxic stress correlates with changes in BBB permeability and ZO-1 localization (51). Bruban et al showed that disorganization of cytoskeletal actin filament models was accompanied by decreased expression of tight junction proteins by the RPE (52).

\section{Inflammatory cytokines induce the destruction of tight junctions}

A number of cytokines have been reported to be upregulated in the plasma of HIV-infected individuals or in plasma treated ex vivo. In vitro, the interaction of HIV or the HIV gp120 envelope with CD4 molecules induces the secretion of tumor necrosis factor (TNF)- $\alpha$, interleukin (IL)-1 and other cytokines (53). Several studies have noted a marked increase in membrane permeability following exposure to vasoactive cytokines, including TNF- $\alpha$, IL- $1 \beta$, interferon- $\gamma$, histamine 65 and growth factors (54). When activated, inflammatory cells initiate the cellular release of free radicals, cytokines and grow th factors (55). HIV-1 Tat is a strong proinflammatory agent that recruits and induces the transendothelial migration of monocytes (56). When Tat was injected into the hippocampi of mice, reductions in the levels of ZO-1 and its continuity were observed, in addition to inflammatory cell accumulation in the choroid plexus (57). It has also been shown that the expression of the occludin promoter is affected by TNF- $\alpha$ or interferon treatment (58). These studies indicate that the expression of HIV genes or proteins may alter the capacity of the cells to secrete important cytokines. Therefore, cytokines are likely to play a vital role in the pathology of HIV-associated complications.

\section{Concluding remarks}

The interplay between HIV-1 and hosts at the BRB is complex. The present review has shown that specific viral genes affect signaling pathways, the expression of enzymes, including MMPs, and cytokines that affect inflammation, which leads to the disruption of tight junctions. These effects are directly caused by HIV-1-associated proteins or HIV-1-induced inflammatory factors. This accumulating damage results in BRB breakdown. Advances in the understanding of HIV-host interactions are likely to be forthcoming as researchers apply effective approaches to their studies of this challenging topic. The present study reveals insights into the molecular mechanisms underlying $\mathrm{BRB}$ regulation and may provide opportunities for the treatment of ocular complications.

\section{Acknowledgments}

The study was supported by a grant from the National Nature Science Foundation of China (no. 81070757).

\section{References}

1. Han Y, Wu N, Zhu W, Li Y, Zuo L, Ye J, Qiu Z, Xie J and Li T: Detection of HIV-1 viruses in tears of patients even under long-term HAART. Aids 25: 1925-1927, 2011.

2. Fujikawa LS, Salahuddin SZ, Palestine AG, Masur H, Nussenblatt RB and Gallo RC: Isolation of human T-lymphotropic virus type III from the tears of a patient with the acquired immunodeficiency syndrome. Lancet 2: 529-530, 1985.

3. Tervo T, Lähdevirta J, Vaheri A, Valle SL and Suni J: Recovery of HTLV-III from contact lenses. Lancet 1: 379-380, 1986.

4. Ablashi DV, Sturzenegger S, Hunter EA, Palestine AG, Fujikawa LS, Kim MK, Nussenblatt RB, Markham PD and Salahuddin SZ: Presence of HTLV-III in tears and cells from the eyes of AIDS patients. J Exp Pathol 3: 693-703, 1987.

5. Pathanapitoon K, Riemens A, Kongyai N, Sirirungsi W, Leechanachai P, Ausayakhun S, Kalinina Ayuso V, Kunavisarut P, de Groot-Mijnes JD and Rothova A: Intraocular and plasma HIV-1 RNA loads and HIV uveitis. Aids 25: 81-86, 2011.

6. González-Scarano F and Martín-García J: The neuropathogenesis of AIDS. Nat Rev Immunol 5: 69-81, 2005.

7. Persidsky $Y$ and Poluektova L: Immune privilege and HIV-1 persistence in the CNS. Immunol Rev 213: 180-194, 2006.

8. De Luca A, Ciancio BC, Larussa D, Murri R, Cingolani A, Rizzo MG, Giancola ML, Ammassari A and Ortona L: Correlates of independent HIV-1 replication in the CNS and of its control by antiretrovirals. Neurology 59: 342-347, 2002.

9. Bai L, Zhang Z, Zhang H, Li X, Yu Q, Lin H and Yang W: HIV-1 Tat protein alter the tight junction integrity and function of retinal pigment epithelium: an in vitro study. BMC Infect Dis 8: 77, 2008.

10. Pomerantz RJ, Kuritzkes DR, de la Monte SM, Rota TR, Baker AS, Albert D, Bor DH, Feldman EL, Schooley RT and Hirsch MS: Infection of the retina by human immunodeficiency virus type I. N Engl J Med 317: 1643-1647, 1987.

11. Abbott NJ, Patabendige AA, Dolman DE, Yusof SR and Begley DJ: Structure and function of the blood-brain barrier. Neurobiol Dis 37: 13-25, 2010.

12. Wislocki GB and Ladman AJ: The demonstration of a blood-ocular barrier in the albino rat by means of the intravitam deposition of silver. J Biophys Biochem Cytol 1: 501-510, 1955.

13. Engelhardt B and Sorokin L: The blood-brain and the blood-cerebrospinal fluid barriers: function and dysfunction. Semin Immunopathol 31: 497-511, 2009.

14. Cunha-Vaz JG: The blood-ocular barriers: past, present, and future. Doc Ophthalmol 93: 149-157, 1997.

15. Fanning AS, Mitic LL and Anderson JM: Transmembrane proteins in the tight junction barrier. J Am Soc Nephrol 10: 1337-1345, 1999.

16. Hawkins BT and Davis TP: The blood-brain barrier/neurovascular unit in health and disease. Pharmacol Rev 57: 173-185, 2005. 
17. Stamatovic SM, Keep RF and Andjelkovic AV: Brain endothelial cell-cell junctions: how to 'open' the blood brain barrier. Curr Neuropharmacol 6: 179-192, 2008.

18. Contreras-Ruiz L, Schulze U, García-Posadas L, Arranz-Valsero I, López-García A, Paulsen F and Diebold Y: Structural and functional alteration of corneal epithelial barrier under inflammatory conditions. Curr Eye Res 37: 971-981, 2012.

19. Morita K, Sasaki H, Furuse M and Tsukita S: Endothelial claudin: claudin-5/TMVCF constitutes tight junction strands in endothelial cells. J Cell Biol 147: 185-194, 1999.

20. Nitta T, Hata M, Gotoh S, Seo Y, Sasaki H, Hashimoto N, Furuse $M$ and Tsukita S: Size-selective loosening of the blood-brain barrier in claudin-5-deficient mice. J Cell Biol 161 653-660, 2003

21. Antonetti DA, Barber AJ, Khin S, Lieth E, Tarbell JM and Gardner TW; Penn State Retina Research Group: Vascular permeability in experimental diabetes is associated with reduced endothelial occludin content: vascular endothelial growth factor decreases occludin in retinal endothelial cells. Diabetes 47: 1953-1959, 1998.

22. Peng S, Adelman RA and Rizzolo LJ: Minimal effects of VEGF and anti-VEGF drugs on the permeability or selectivity of RPE tight junctions. Invest Ophthalmol Vis Sci 51: 3216-3225, 2010.

23. Harhaj NS and Antonetti DA: Regulation of tight junctions and loss of barrier function in pathophysiology. Int $\mathrm{J}$ Biochem Cell Biol 36: 1206-1237, 2004

24. Feldman GJ, Mullin JM and Ryan MP: Occludin: structure, function and regulation. Adv Drug Deliv Rev 57: 883-917, 2005.

25. Erickson KK, Sundstrom JM and Antonetti DA: Vascular permeability in ocular disease and the role of tight junctions. Angiogenesis 10: 103-117, 2007.

26. Behzadian MA, Windsor LJ, Ghaly N, Liou G, Tsai NT and Caldwell RB: VEGF-induced paracellular permeability in cultured endothelial cells involves urokinase and its receptor. FASEB J 17: 752-754, 2003.

27. Chehade JM, Haas MJ and Mooradian AD: Diabetes-related changes in rat cerebral occludin and zonula occludens-1 (ZO-1) expression. Neurochem Res 27: 249-252, 2002.

28. Fischer S, Wobben M, Marti HH, Renz D and Schaper W: Hypoxia-induced hyperpermeability in brain microvessel endothelial cells involves VEGF-mediated changes in the expression of zonula occludens-1. Microvasc Res 63: 70-80, 2002.

29. Jin M, Barron E, He S, Ryan SJ and Hinton DR: Regulation of RPE intercellular junction integrity and function by hepatocyte growth factor. Invest Ophthalmol Vis Sci 43: 2782-2790, 2002.

30. Lum H and Malik AB: Regulation of vascular endothelial barrier function. Am J Physiol 267: L223-L241, 1994.

31. Davidson DC, Hirschman MP, Sun A, Singh MV, Kasischke K and Maggirwar SB: Excess soluble CD40L contributes to blood brain barrier permeability in vivo: implications for HIV-associated neurocognitive disorders. PloS One 7: e51793, 2012

32. Albini A, Benelli R, Presta M, Rusnati M,Ziche M, Rubartelli A, Paglialunga G, Bussolino F and Noonan D: HIV-tat protein is a heparin-binding angiogenic growth factor. Oncogene 12: 289-297, 1996

33. Wennerberg K, Rossman KL and Der CJ: The Ras superfamily at a glance. J Cell Sci 118: 843-846, 2005.

34. Cohen AW, Hnasko R, Schubert W and Lisanti MP: Role of caveolae and caveolins in health and disease. Physiol Rev 84: $1341-1379,2004$

35. Zhong Y, Zhang B, Eum SY and Toborek M: HIV-1 Tat triggers nuclear localization of ZO-1 via Rho signaling and cAMP response element-binding protein activation. J Neurosci 32 143-150, 2012

36. Lin S, Wang XM, Nadeau PE and Mergia A: HIV infection upregulates caveolin 1 expression to restrict virus production. J Virol 84: 9487-9496, 2010.

37. Nag S, Venugopalan R and Stewart DJ: Increased caveolin-1 expression precedes decreased expression of occludin and claudin-5 during blood-brain barrier breakdown. Acta Neuropathol 114: 459-469, 2007.

38. Clark IM, Swingler TE, Sampieri CL and Edwards DR: The regulation of matrix metalloproteinases and their inhibitors. Int J Biochem Cell Biol 40: 1362-1378, 2008

39. Feng S, Cen J, Huang Y, Shen H, Yao L, Wang Y and Chen Z Matrix metalloproteinase-2 and -9 secreted by leukemic cells increase the permeability of blood-brain barrier by disrupting tight junction proteins. PloS One 6: e20599, 2011.
40. Yang Y, Estrada EY, Thompson JF, Liu W and Rosenberg GA: Matrix metalloproteinase-mediated disruption of tight junction proteins in cerebral vessels is reversed by synthetic matrix metalloproteinase inhibitor in focal ischemia in rat. J Cereb Blood Flow Metab 27: 697-709, 2007.

41. Gurney KJ, Estrada EY and Rosenberg GA: Blood-brain barrier disruption by stromelysin-1 facilitates neutrophil infiltration in neuroinflammation. Neurobiol Dis 23: 87-96, 2006.

42. Reijerkerk A, Kooij G, van der Pol SM, Khazen S, Dijkstra CD and de Vries HE: Diapedesis of monocytes is associated with MMP-mediated occludin disappearance in brain endothelial cells. FASEB J 20: 2550-2552, 2006.

43. McCoig C, Castrejon MM, Saavedra-Lozano J, Castano E, Baez C, Lanier ER, Saez-Llorens X and Ramilo O: Cerebrospinal fluid and plasma concentrations of proinflammatory mediators in human immunodeficiency virus-infected children. Pediatr Infect Dis J 23: 114-118, 2004.

44. Sporer B, Paul R, Koedel U, Grimm R, Wick M, Goebel FD and Pfister HW: Presence of matrix metalloproteinase-9 activity in the cerebrospinal fluid of human immunodeficiency virus-infected patients. J Infect Dis 178: 854-857, 1998.

45. Persidsky Y, Limoges J, Rasmussen J, Zheng J, Gearing A and Gendelman HE: Reduction in glial immunity and neuropathology by a PAF antagonist and an MMP and TNFalpha inhibitor in SCID mice with HIV-1 encephalitis. J Neuroimmunol 114: 57-68, 2001.

46. Louboutin JP and Strayer DS: Blood-brain barrier abnormalities caused by HIV-1 gp120: mechanistic and therapeutic implications. ScientificWorldJournal 2012: 482575, 2012.

47. Xu R, Feng X, Xie X, Zhang J, Wu D and Xu L: HIV-1 Tat protein increases the permeability of brain endothelial cells by both inhibiting occludin expression and cleaving occludin via matrix metalloproteinase-9. Brain Res 1436: 13-19, 2012.

48. Giebel SJ, Menicucci G, McGuire PG and Das A: Matrix metalloproteinases in early diabetic retinopathy and their role in alteration of the blood-retinal barrier. Lab Invest 85: 597-607, 2005.

49. Lai CH, Kuo KH and Leo JM: Critical role of actin in modulating BBB permeability. Brain Res Brain Res Rev 50: 7-13, 2005.

50. Schreibelt G, Kooij G, Reijerkerk A, van Doorn R, Gringhuis SI, van der Pol S, Weksler BB, Romero IA, Couraud PO, Piontek J, et $a l$ : Reactive oxygen species alter brain endothelial tight junction dynamics via RhoA, PI3 kinase, and PKB signaling. FASEB J 21: 3666-3676, 2007

51. Hicks K, O'Neil RG, Dubinsky WS and Brown RC: TRPC-mediated actin-myosin contraction is critical for BBB disruption following hypoxic stress. Am J Physiol Cell Physiol 298: C1583-C1593, 2010.

52. Bruban J, Glotin AL, Dinet V, Chalour N, Sennlaub F, Jonet L, An N, Faussat AM and Mascarelli F: Amyloid-beta(1-42) alters structure and function of retinal pigmented epithelial cells. Aging Cell 8: 162-177, 2009.

53. D'Addario M, Wainberg MA and Hiscott J: Activation of cytokine genes in HIV-1 infected myelomonoblastic cells by phorbol ester and tumor necrosis factor. J Immunol 148: 1222-1229, 1992.

54. Mohammad G, Siddiquei MM, Othman A, Al-Shabrawey M and Abu El-Asrar AM: High-mobility group box-1 protein activates inflammatory signaling pathway components and disrupts retinal vascular-barrier in the diabetic retina. Exp Eye Res 107: 101-109, 2013.

55. Keshari RS, Jyoti A, Dubey M, Kothari N, Kohli M, Bogra J, Barthwal MK and Dikshit M: Cytokines induced neutrophil extracellular traps formation: implication for the inflammatory disease condition. PloS One 7: e48111, 2012.

56. Weiss JM, Nath A, Major EO and Berman JW: HIV-1 Tat induces monocyte chemoattractant protein-1-mediated monocyte transmigration across a model of the human blood-brain barrier and up-regulates CCR 5 expression on human monocytes. J Immunol 163: 2953-2959, 1999.

57. Pu H, Tian J, Andras IE, Hayashi K, Flora G, Hennig B and Toborek M: HIV-1 Tat protein-induced alterations of ZO-1 expression are mediated by redox-regulated ERK $1 / 2$ activation. J Cereb Blood Flow Metab 25: 1325-1335, 2005.

58. Mankertz J, Tavalali S, Schmitz H, Mankertz A, Riecken EO, Fromm $M$ and Schulzke JD: Expression from the human occludin promoter is affected by tumor necrosis factor alpha and interferon gamma. J Cell Sci 113: 2085-2090, 2000. 